\title{
Making pathogens sociable: The emergence of high relatedness through limited host invasibility
}

This article has been corrected since Advance Online Publication and a corrigendum is also printed in this issue

\author{
Edwin van Leeuwen ${ }^{1}$, Sarah O’Neill ${ }^{1}$, Andrew Matthews ${ }^{1,2}$ and Ben Raymond ${ }^{1,2}$ \\ ${ }^{1}$ School of Biological Sciences, Royal Holloway University of London, Egham, UK and ${ }^{2}$ Department of Life \\ Sciences, Imperial College London, Silwood Park campus, Ascot, UK
}

\begin{abstract}
Cooperation depends upon high relatedness, the high genetic similarity of interacting partners relative to the wider population. For pathogenic bacteria, which show diverse cooperative traits, the population processes that determine relatedness are poorly understood. Here, we explore whether within-host dynamics can produce high relatedness in the insect pathogen Bacillus thuringiensis. We study the effects of host/pathogen interactions on relatedness via a model of host invasion and fit parameters to competition experiments with marked strains. We show that invasibility is a key parameter for determining relatedness and experimentally demonstrate the emergence of high relatedness from well-mixed inocula. We find that a single infection cycle results in a bottleneck with a similar level of relatedness to those previously reported in the field. The bottlenecks that are a product of widespread barriers to infection can therefore produce the population structure required for the evolution of cooperative virulence.
\end{abstract}

The ISME Journal (2015) 9, 2315-2323; doi:10.1038/ismej.2015.111; published online 30 June 2015

\section{Introduction}

Pathogenic bacteria appear to be particularly dependent upon cooperation (West et al., 2006). They rely upon the secretion of a wide range of signals and virulence factors such as pore-forming toxins, siderophores and quorum-sensing molecules (West and Buckling, 2003; Brockhurst et al., 2006; Diggle et al., 2007; Raymond et al., 2012). While many retain the private benefits of virulence factors by injecting them locally into host cells using type IV and type III secretion systems (Bomberger et al., 2009), a large number of pathogens release virulence factors that act at a distance (West and Buckling, 2003; SchmidHempel and Frank, 2007; Bomberger et al., 2009; Raymond et al., 2012). By releasing metabolites into extracellular space, the benefits of these virulence factors are exploitable by neighbours. These shared products have been commonly described as 'public goods' (Sachs et al., 2004). A key issue for the persistence of this form of cooperation is its vulnerability to exploitation by 'cheater' mutants that reap the benefits of investment by their neighbours without paying the costs themselves

Correspondence: E van Leeuwen, School of Biological Sciences, Royal Holloway University of London, Egham Hill, Egham, TW200EX, UK.

E-mail: edwinvanl@gmail.com

Received 22 December 2014; revised 16 April 2015; accepted 19 May 2015; published online 30 June 2015
(Frank, 1998). High relatedness, which increases the inclusive fitness of cooperators, can prevent cooperative strains from being out-competed by cheater mutants (Hamilton, 1964; Griffin et al., 2004; Diggle et al., 2007; Gilbert et al., 2007). However, the population structure of pathogens has rarely been resolved at a fine enough scale to allow ecologically relevant calculations of relatedness (but see Raymond et al., 2012).

Explaining how high relatedness arises is an area of much interest in microbial social biology (Nadell et al., 2010 and 2013; Mitri et al., 2011; Damore and Gore, 2012). Mechanisms such as kin discrimination promote high relatedness and social interactions among kin in higher organisms (West et al., 2007; Gilbert et al., 2012). In bacteria, kin-discrimination mechanisms are largely unknown. However, bacteria may be able to alter the relatedness of their local environment via antagonistic secretions such as bacteriocins, which can selectively impose mortality on non-kin (Garbutt et al., 2011). Horizontal gene transfer can also actively increase relatedness in bacterial populations, as bacteria can 'infect' neighbouring cells with genes required for cooperative activity (Rankin et al., 2011). However, the broad impact of antagonism and horizontal gene transfer on bacterial population structure is still largely unknown. In general, stochastic processes may be very important in microorganisms. Stochastic sorting of different genotypes can occur during dispersal and 
during the division and growth of bacteria in biofilms. This can, theoretically at least, lead to population bottlenecks and the spatial structure required to maintain the production of public goods (Nadell et al., 2010 and 2013; Korolev et al., 2011; Mitri et al., 2011; Datta et al., 2013; Momeni et al., 2013). Although spatial structure in biofilms clearly is important for maintaining high relatedness on a local scale, it is unclear how this translates to the maintenance of relatedness during transmission of bacteria from host to host. Here, we will explore the role of stochasticity during the invasion of hosts by bacterial pathogens and its impact on relatedness.

Mixed infections by genetically diverse bacteria of the same species are believed to happen less often than single strain infections in humans, but still happen in, on average, 21.7\% of infections (Balmer and Tanner, 2011), and some studies show an even higher rate (Levert et al., 2010). A number of studies have shown that the progress of infection through hosts is associated with population bottlenecks and the loss of pathogen diversity (Moxon and Murphy, 1978; Brown et al., 2006; Grant et al., 2008; Plaut et al., 2012; Prajsnar et al., 2012), so that a plausible general explanation for the low diversity (and therefore high relatedness) of bacterial parasites in hosts is the existence of strong bottlenecks during colonisation. However, many of these previous examples involve infections generated artificially in model hosts (Grant et al., 2008; Prajsnar et al., 2012), or diseases such as inhalational anthrax and meningitis (Moxon and Murphy, 1978; Plaut et al., 2012), which do not represent a normal or successful mode of transmission for these pathogens. The importance of social interactions, and hence changes in diversity, has also not been explored for any of these pathogens.

It is therefore unclear how natural population processes, over the entire natural cycle of infection, affect relatedness at a spatial scale that is meaningful for microbial social interactions and on-going transmission. For Bacillus thuringiensis, a widespread pathogen of invertebrates (Raymond et al., 2010a), the importance of social interactions and high relatedness is very clear. These bacteria produce proteinaceous crystal (Cry) toxins at sporulation that determine virulence and host-range and that are required for pathogenicity (Schnepf et al., 1998). Cry toxin production is cooperative at the scale of a single host, and natural populations are characterised by high levels of relatedness (Raymond et al., 2012), although the population processes leading to high relatedness have not yet been clearly established. Here, we hypothesise that the successive bottlenecks involved in transmission can generate levels of relatedness (at the host scale) similar to that seen in field populations of the entomopathogen, B. thuringiensis (Raymond et al., 2012). Thus, general within-host population processes should preserve the high levels of population structure required for effective cooperation and maintenance of virulence.

\section{Materials and methods}

We explored experimentally and theoretically whether within-host population dynamics were sufficient to explain observed high levels of relatedness at the level of the host. We specifically considered the dynamics of mixed infections and explored four parameters that can affect the structuring of co-infecting genotypes. The theoretical model was parameterised and tested using in vivo experiments with competing genotypes of $B$. thuringiensis, marked with different spontaneous antibiotic resistance (to rifampicin and nalidixic acid). Insects were inoculated orally by feeding on droplets containing a well-defined dose (300 cfu (colony-forming unit) in total, approximately LD90) with varying proportions of each genotype.

\section{Model derivation}

The probability density function of the final strain abundance was derived based on an invasion-growth model. Time to successful invasion for each strain was exponentially distributed:

$f_{t_{0}}\left(t_{0} \mid \Lambda_{i}\right)=\left\{\begin{array}{ll}0, & t_{0}<t \\ \Lambda_{i} e^{-\Lambda_{i} t_{0}}, & t_{0} \geqslant t\end{array}\right.$,

with $\Lambda_{i}$ the successful invasion rate of strain $i$ and $t_{0}$ the time of invasion. After invasion, each strain grew deterministically and at a constant rate. The density of strain $i\left(n_{i}\right)$ at time $t$ can then be defined as follows:

$n_{i}(t)= \begin{cases}0, & t_{0}>t \\ e^{\gamma_{i}\left(t-t_{0}\right)}, & t_{0} \leqslant t\end{cases}$

with $\gamma_{i}$, the growth rate within the host. When considering two strains ( $a$ and $b$ ), it is possible to rescale the parameters without loss of generality as follows: the number of infectious pathogens successfully entering a host per unit time $\left(\lambda=\left(\Lambda_{a}+\Lambda_{b}\right) / \gamma_{a}\right)$, the proportion of the strain $a$ in the inoculum of coinvading genotypes (between 0 and 1;p), the relative growth rate of strain $b\left(\gamma=\gamma_{b} / \gamma_{a}\right)$ and the time since infection $\left(\tau=\gamma_{a} t\right)$. On the basis of these assumptions, we were able to derive the full probability distribution of the proportion $(x)$ of the two strains after a certain time $\left(f_{x}(x \mid p, \lambda, \gamma, \tau)\right.$; see Supplementary Appendix S1 in Supporting Information for more details). The final proportion of each strain was highly dependent on which strain invaded first, because that strain has more time to grow and become dominant. We also explored how sensitive the model was to the choice of growth model and show that the results will hold up for a LotkaVolterra competition model as long as both strains are sufficiently similar (see Supplementary Appendix S2 in the Supporting Information).

Relatedness was used as a measure of the population structure after infection. We calculate the relatedness coefficient as defined by Queller and Goodnight, 1989 from our model to further explore 
the effect of the infection barrier. The resulting relatedness coefficient is between 0 and 1 . Relatedness can then be interpreted as the probability to pick (with replacement) two individuals of the same strain from one subpopulation (insect) minus the probability to pick two individuals of the same type from the population as a whole, divided by 1 minus the probability to pick two individuals of the same type from the populations as a whole. If the subpopulation was dominated by one strain, but not the whole population, then the relatedness is close to 1. Note though, that if the population as a whole is also dominated by the same strain, then relatedness coefficient is low, because the genetic similarity in the sub-population reflects that across the entire population. We calculated the relatedness using the most likely parameter values. See Supplementary Appendix S1 in Supporting Information for more details on the model derivation and fitting.

\section{Model validation}

A likelihood-based method was used to find the most likely parameter values given the data. The likelihood of different parameter values was calculated based on the abundance counts collected during the experiment. We then used an adaptive Differential Evolution-based algorithm to find the parameters which resulted in a maximum likelihood value (Brest et al., 2006). Next, we bootstrapped the data to find the upper and lower limits of the parameter values using a $95 \%$ confidence interval. The relative importance of the underlying processes could be inferred from these results.

We compared the findings of the model presented above with an alternative model, where we assumed that the main limiting process in the infection was the survival of the inoculum to initial infection. In this case, a low survival rate would lead to large variation in the final proportion $(x)$, because the final proportion is dependent on the number of bacteria of each strain to survive till successful founding of the infection. Comparing the results of the fit of our model with this alternative model was performed to quantify whether our model provides a better explanation of the observed experimental results. See Supplementary Appendix S3 in the Supporting Information for more details on this alternative model.

\section{Experimental design}

Spontaneous antibiotic resistance mutants of B. thuringiensis kurstaki HD-1 were isolated from the commercial biopesticide preparation, DiPel WP (Valent Biosciences, Libertyville, IL, USA), by plating high numbers of cells $\left(10^{8}+\mathrm{cfu}\right)$ on $100 \mu \mathrm{g}$ $\mathrm{ml}^{-1}$ rifampicin or $15 \mu \mathrm{g} \mathrm{ml}^{-1}$ nalidixic acid. Antibiotic-resistant mutants with reduced fitness costs were isolated after one to three rounds of host passage in Plutella xylostella (Garbutt et al., 2011), and identified by rapid growth on selective plates. Lack of cross resistance between two mutants R4K1 Rif $^{R}$ and P1G Nal ${ }^{R}$ was confirmed by thickly streaking sporulated cultures across LB agar plates containing appropriate antibiotics. Although these strains had a recent common ancestor, we also investigated the pleiotropic consequences of resistance mutations with several life history experiments. These included pathogenicity assays at three doses with 30-40 insects per dose, as per the droplet assay described below and measures of spore productivity in infections with a single strain (with at least $N=40$ cadavers per strain).

Sporulated cultures of both strains were produced by growing dense lawns of bacteria on HCO sporulation media (Lecadet et al., 1980) at $30^{\circ} \mathrm{C}$ for 1 week. Spores and Cry toxins were recovered from plates and washed twice in sterile saline $(0.85 \% \mathrm{NaCl})$, before being diluted into $10 \mathrm{ml}$ of saline and stored at $-20^{\circ} \mathrm{C}$ in $0.5 \mathrm{ml}$ aliquots. Defrosted spores were enumerated by plating serial dilutions; counts were made within $48 \mathrm{~h}$ of infecting insects.

P. xylostella larvae were reared on artificial diet (without antibiotics) as described previously (Raymond et al., 2009). Late second instars were infected with $300 \mathrm{cfu}$ of $B t$ in droplet assays. The final droplet mix contained $10 \mathrm{~mm}$ sucrose, $7.5 \% \mathrm{v} / \mathrm{v}$ green food dye (Dr Oetker, Bielefield, Germany) and $0.4 \% \mathrm{w} / \mathrm{v}$ agar (Oxoid, Basingstoke, UK), and 40\% v/v cabbage extract (filtered liquid from boiled cabbages). The cabbage juice, sucrose and food dye were filtersterilised before being used to dilute the spores; this mixture was then combined (50:50) with molten $0.8 \%$ agar at $60^{\circ} \mathrm{C}$. The resultant inoculum (with a final concentration of $300 \mathrm{cfu}^{-1}$ ) was briefly held at $50^{\circ} \mathrm{C}$ in a heat block (for no more than $10 \mathrm{~min}$ ) while $1 \mu \mathrm{l}$ droplets were dispensed into each well of 48-well plates using pre-warmed pipette tips. A single larva was added to each well, and plates were tightly sealed with damp tissue paper. Larvae were allowed to feed for $18 \mathrm{~h}$. When droplets were at least $75 \%$ consumed and green dye was visible within larvae (Figure 1), insects were moved onto artificial diet for 5 days. Mortality rates were typically greater than $90 \%$ in these assays. Cadavers were transferred to $2 \mathrm{ml}$ homogenisation tubes containing $10 \mu \mathrm{l}$ of sterile water and incubated at $30^{\circ} \mathrm{C}$ for at least 7 days. This procedure ensures that all Bt within cadavers had fully sporulated before homogenisation and final enumeration. Before homogenisation, $500 \mu \mathrm{l}$ of sterile saline $(0.85 \% \mathrm{NaCl})$ was added to each homogenisation tube and cadavers were pasteurised at $65^{\circ} \mathrm{C}$ for $20 \mathrm{~min}$ to kill any remaining vegetative cells. Cadavers were homogenised in a beadbeater (Qiagen Tissue Lyzer, Manchester, UK) using 4-mm diameter steel ball bearings. Homogenates were serially diluted in saline and plated out on rifampicin $\left(100 \mu \mathrm{g} \mathrm{ml}^{-1}\right)$ and nalidixic acid (15 $\mu \mathrm{g} \mathrm{ml}^{-1}$ ) LB agar plates in $15 \mu \mathrm{l}$ droplets before being incubated overnight at $30^{\circ} \mathrm{C}$. As a general rule, 


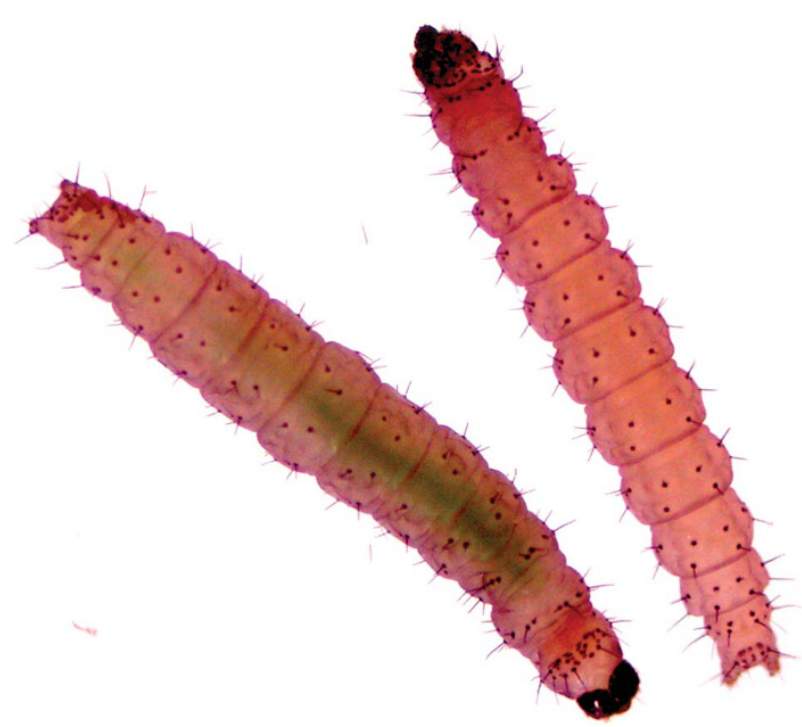

Figure 1 Second instar diamondback moth larvae were infected with $B$. thuringiensis spores in agarose droplets containing green food dye. This allowed us to readily identify insects that had consumed a defined dose.

dilutions providing colony counts between 5 and 50 per droplet were included in the data set. Replicated droplets counts (within cadavers) confirmed the repeatability of this enumeration technique.

\section{Results}

The model predicts the exact probability of finding a certain final proportion of competing bacteria in a host given different parameter values (Figure 2). To better understand the implications of the possible probability density functions, we classified them according to three different attributes. Firstly, the final population can be 'well-mixed', where there is a high probability of the abundances of the two strains being about equal in each cadaver. In contrast, the population can also be 'bimodal', where there is a high probability that one strain will dominate the other strain in a cadaver. Although, in this case, each sample is dominated by one strain, both strains have an equal probability of becoming the dominating strain. Finally, the population can be 'skewed', where most cadavers are dominated by one particular strain, that is, the population as a whole is dominated by one strain. Next, we studied in detail how the different parameters influence these different attributes of the probability density function and the resulting relatedness of the population as a whole.

Relatedness is high when the genetic similarity within host communities is high relative to the population at large (Queller and Goodnight, 1989). Bimodality in part reflects the predicted relatedness of pathogens after one round of infection, as under highly bimodal distributions competing genotypes will be assorted into different cadavers. We measured the bimodality of the probability density function by

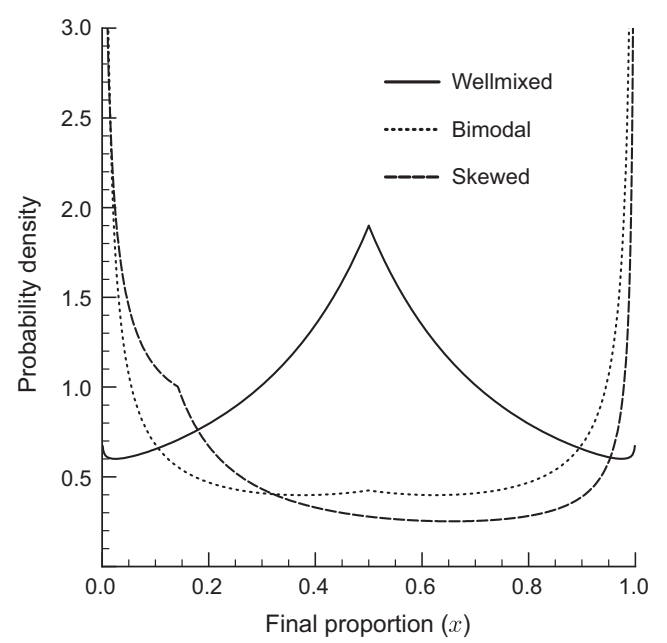

Figure 2 Three typical outcomes of the model, which can be classified as follows: (i) well-mixed, i.e., there is a high probability of finding both strains at roughly equal abundances, (ii) bimodal, where one strain dominates the other, but both strains have an equal probability of being the dominant strain and (iii) skewed, where one strain is more likely to be the abundant strain.

calculating the coefficient of bimodality, which is a function of the third and fourth moment of the distribution and ranges between 0 and 1 (Ellison, 1987). Low invasion rates $(\lambda)$ resulted in a high coefficient of bimodality and therefore in high relatedness (Figures 3a and c). In contrast, increases in the difference between the growth rates $(\gamma)$ or the difference in initial abundance in the inoculum ( $p$ ) also result in high bimodality, but have a less pronounced effect on relatedness, because they also increase skewness in the population (Figures $3 \mathrm{~b}$ and c). Skewness ensures that a single strain dominates both the within- and between-host communities and therefore has a less pronounced effect on relatedness. These results explain why changes in the invasion rate have an especially profound effect on the population structure and relatedness

Mixed infections in insect hosts using near isogenic $B$. thuringiensis strains revealed strongly bimodal patterns of genotype frequencies in infectious spores produced at the end of infection (Figures 4a and c), a pattern consistent with low invasion rates. Maximum likelihood-based model fitting produced a close fit between the theoretical model and each of three independent experiments initiated with different genotype frequencies. Using the maximum likelihood, we calculated the Akaike Information Criterion (AIC; Akaike, 1973) and compared this model with an alternative model, which assumed that the main cause of this variation is due to small founding populations. We found essentially no empirical support for the alternative model when compared with our model (Burnham and Anderson, 2002; see Supplementary Appendix S3 in the Supplementary Information for more details). Results were consistent for all data fitted simultaneously and for the three independent data 

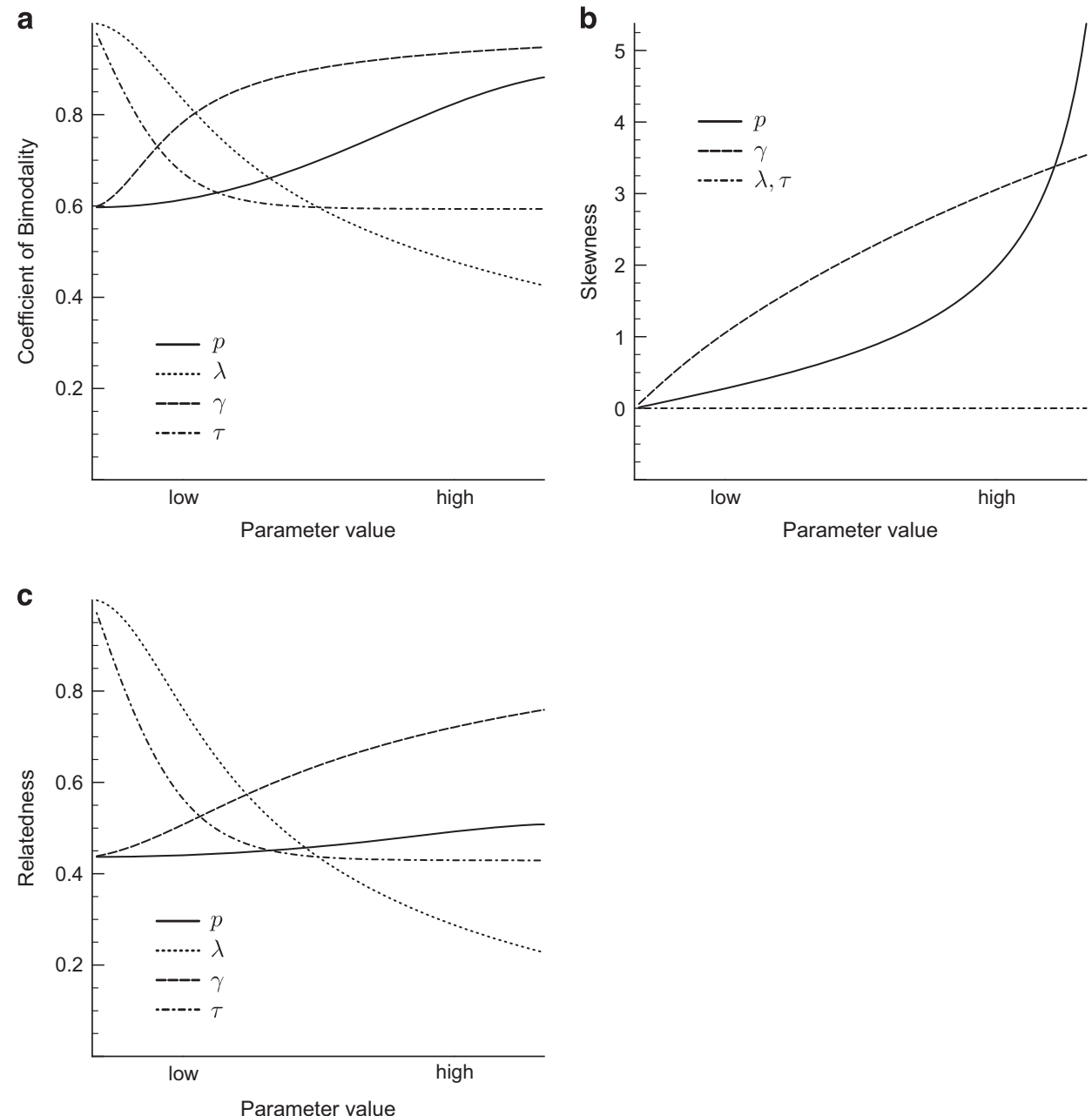

Figure 3 Effect of different parameter values on: (a) bimodality, (b) skewness and (c) relatedness. Under these parameter values, skewness is not affected by the relative invasion rate or time since infection, so both parameters are represented by the same line in b. Default parameter values used: proportion in the inoculum $(P=0.5)$, relative invasion rate $(\lambda=1)$ and time since infection $(\tau=10)$.

sets. Parameterisation of the model in the three experiments was also consistent; in each case, the relative growth rates were similar (Table 1), and the overall relative growth rate of the $\mathrm{Nal}^{\mathrm{R}}$ strain was 1.09, indicating that the fitness of the two mutants was very similar. Given the estimated parameter values, relatedness is consistently high (0.7-0.8) regardless of inoculum proportion (Figure $4 \mathrm{~d}$ ). This highlights that the low infection rates measured in this system result in high relatedness even when starting from a well-mixed population in the inoculum.

More direct calculations of relative fitness validate the results of the model fitting. The change in proportion of a particular mutant $\left(\mathrm{P} 1 \mathrm{G} \mathrm{Nal}{ }^{\mathrm{R}}\right)$ can be used to calculate fitness (Ross-Gillespie et al., 2007). After dividing this estimate by $\log _{2}$ (total spore counts), we can estimate the relative growth rate per generation at $1.08 \quad(N=332$ larvae, $95 \%$ confidence intervals 1.06-1.10). Additional life history differences confirmed that there exist small, but significant differences in pathogenicity $\left(\chi^{2}=7.43\right.$, df $=1, P=0.0064$, Supplementary Information S4) which would equate to approximately a $15 \%$ difference in mortality rates at our experimental dose. The faster growing and less pathogenic strain $\left(\mathrm{Nal}^{\mathrm{R}}\right)$ also produced slightly more spores per insect host in single strain infections $\left(\mathrm{F}_{1,83}=7.87\right.$, $P=0.0063$; Supplementary Information S4).

\section{Discussion}

Here, we have shown that the simple natural process of infection can produce strong between-host heterogeneity in pathogen distribution from an initially well-mixed inoculum containing competing genotypes. Clonal mixing, or low relatedness, in reservoirs of naturally distributed bacteria therefore presents no real challenge to socially based explanations for maintenance of secreted virulence factors with moderate fitness costs, because a single 

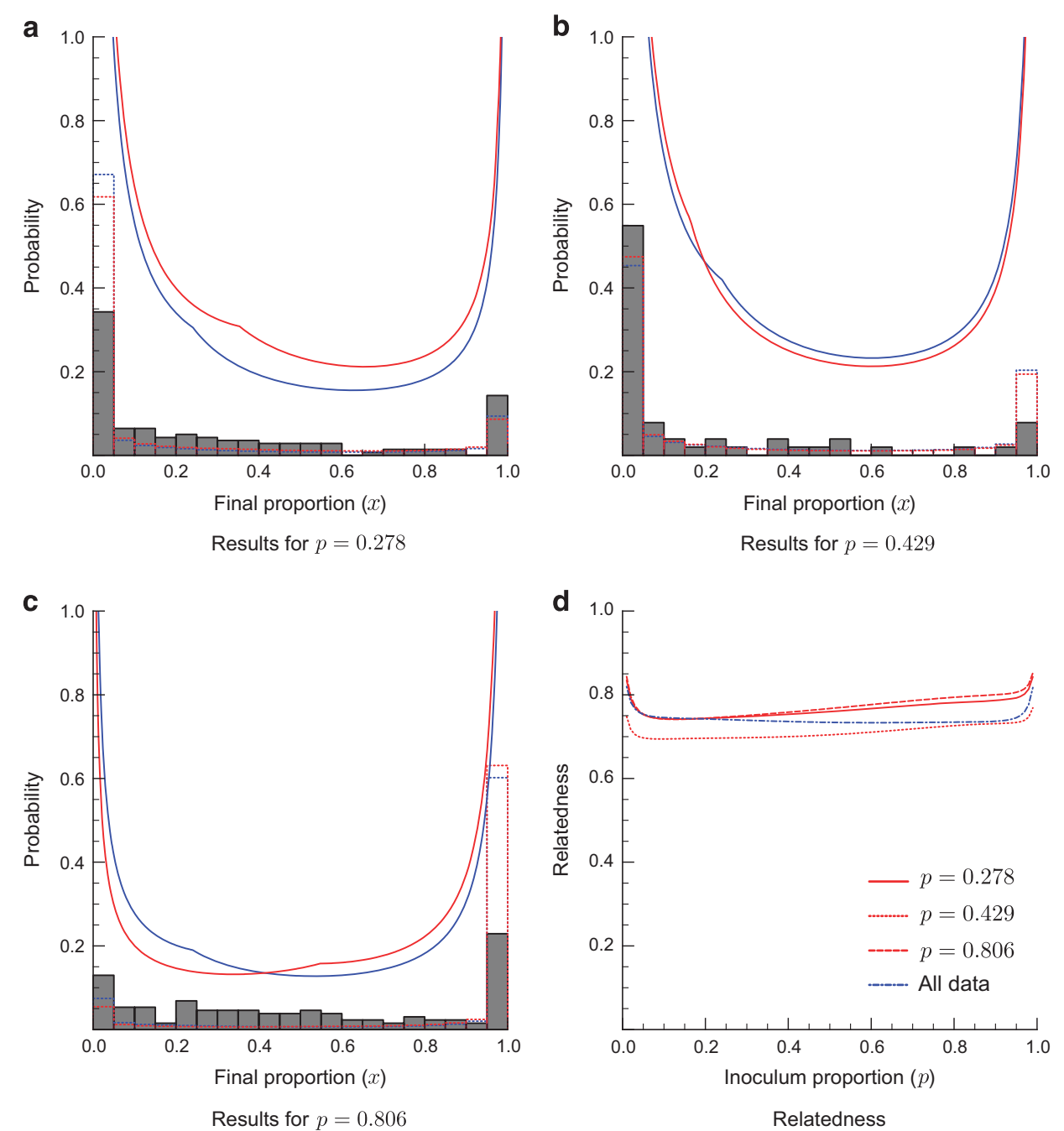

Figure 4 Model fitting results. Blue corresponds to the model when fitted to all the data, while red corresponds to the model fitted to the data with that proportion in the inoculum. $(\mathbf{a}-\mathbf{c})$ The lines show the predicted probability density function. The dotted lines show the same probability distribution, but taken over intervals. This makes visual comparison between the model and the data easier. The grey histogram shows the data. Each sub-figure shows the data and results grouped by inoculum proportion. (d) Relatedness as dependent on the initial inoculum population using the parameters from the fitted models.

Table 1 Results of the parameter fitting

\begin{tabular}{lcccc}
\hline Inoculum $(p)$ & Parameter & Max $L$ & 5th percentile & 95th percentile \\
\hline All data & $\lambda$ & 0.388255 & 0.369545 & 0.433066 \\
All data & $\gamma$ & 1.08953 & 1.04781 & 1.12001 \\
All data & $\tau$ & 12.8511 & 12.3539 & 12.8601 \\
0.278 & $\lambda$ & 0.45007 & 0.403914 & 1.03256 \\
0.278 & $\gamma$ & 1.04707 & 12.3224 & 1.09277 \\
0.278 & $\tau$ & 12.8601 & 0.353042 & 12.8601 \\
0.429 & $\lambda$ & 0.398937 & 1.08419 & 19.546645 \\
0.429 & $\gamma$ & 1.13228 & 10.9993 & 12.3659 \\
0.429 & $\tau$ & 12.3659 & 0.37108 & 0.487739 \\
0.806 & $\lambda$ & 0.401246 & 0.963852 & 1.03481 \\
0.806 & $\gamma$ & 0.9846 & 11.9057 & 12.5233 \\
\hline
\end{tabular}

We show the maximum likelihood parameter value and the uncertainty in the parameter value. The percentile interval has been measured using a resampling approach. Results between the different data sets are consistent, with the invasion rate between 0.35 and 0.55 . Relative growth rates refer to the growth of the $\mathrm{Nal}^{\mathrm{R}}$ strain/ $\mathrm{Rif}^{\mathrm{R}}$ and are close to 1; the time of measurement is between 10 and 13 . 
round of infection can produce the high levels of relatedness required to maintain cooperation. Mixed infections of pathogenic bacteria are relatively infrequent in many species infecting human hosts (Balmer and Tanner, 2011). Rarity of multiple infections can be explained by bottlenecks in the host colonisation process as well as barriers to super-infection, the invasion of a host already colonised. For example, Staphylococcus aureus actively prevents super-infection in a rat model (Margolis et al., 2010), and this species has relatively low rates of mixed infection $(<10 \%)$ (Cespedes et al., 2005; Balmer and Tanner, 2011). Where there are no barriers to super-infection, as in Haemophilus influenzae (Margolis et al., 2010), levels of reported mixed infections are correspondingly higher (53\%) (Balmer and Tanner, 2011).

It could be argued that strong bottlenecks are a feature of infection processes in a small invertebrate host. However, the spatial scale at which social interactions occur among microbes can be even smaller (Slater et al., 2008) and several pathogens display patterns of declining diversity as infections proceed (Moxon and Murphy, 1978; Brown et al., 2006; Grant et al., 2008; Plaut et al., 2012; Prajsnar et al., 2012). Low diversity may even be the product of strategies to maintain very low densities within phagocytes to promote immune evasion (Prajsnar et al., 2012). In this study, the spatial scale of study is appropriate for the major virulence factors (Cry toxins) of our chosen pathogen, because cooperation and cheating based on these solubilised toxins occurs in the midgut of single insects (Raymond et al., 2012). Insect cadavers are a suitable spatial scale for assessing relatedness because of their importance for spore production and ongoing disease transmission, which can occur orally via cannibalism or consumption of patches of foliage contaminated by cadavers (Knell et al., 1996; Raymond et al., 2010a, 2012). A caveat here is that transmission can also occur indirectly, when bacteria from a soil reservoir colonise plants (Raymond et al., 2010b). Note also, that infections in this study used a relatively high dose of inoculum, capable of killing $80-90 \%$ of hosts. This density of spores $\left(>10^{2} \mathrm{cfu} \mathrm{cm}^{2}\right)$ is rare in natural environments (Collier et al., 2005), and low dose infections may further reduce invasibility and increase relatedness (Moxon and Murphy, 1978). Despite this expected difference in doses in the field and the laboratory, measurements of population structure in this study were high and comparable with field measurements of relatedness at the Cry toxin locus of B. thuringiensis in the field (Raymond et al., 2012).

It has been argued that fluctuating population structure poses a challenge to the generality of inclusive fitness theory in microbes (Damore and Gore, 2012). The reasoning is that real-world complexities such as non-linear fitness benefits and changes in relatedness are likely to lead to dynamical fluctuations in $b$, the benefit deriving from cooperation. This makes the 'disentangling of fitness effects from population structure often impossible' (Damore and Gore, 2012). Our data suggest that this problem is not as severe as they suggest. We have previously made some attempt to link population processes to the maintenance of cooperation (Raymond et al., 2012) and would argue that population dynamic processes can act to stabilise relatedness in this pathogen. In the field, relatedness at the locus of Cry toxin production in $B$. thuringiensis can fluctuate within a single patch, but is very stable in the whole population, despite variation in bacterial density and the overall proportion of toxin producers (Raymond et al., 2012). In this study, population bottlenecks ensure that relatedness at nearly neutral loci remained high, despite variation in the initial frequencies of competitors. In the field, similar bottlenecks in infection and transmission (Zhou et al., 2014) or in the colonisation of plants from spore reservoirs in the soil (Raymond et al., 2010b) may also stabilise high relatedness.

Previous observations of extreme bottlenecks during infection have been interpreted as evidence for the ability of bacteria to act independently during infection, as infections appear to be established from single cells, in some circumstances (Moxon and Murphy, 1978; Rubin, 1987). The 'independent action hypothesis' proposes that the probability of infection resulting from a single cell is independent of the presence of conspecifics (Druett, 1952). The independent action hypothesis has widespread importance in epidemiological modelling and risk assessment (Haas, 1983; Buchanan et al., 2009). Bottlenecks have therefore been interpreted as being evidence which supports the independent action hypothesis (Moxon and Murphy, 1978; Rubin 1987). However, the fact that pathogen populations pass through clonal or near-clonal bottlenecks does not mean that cells are acting independently. Shared exploitation of public goods is critical in the virulence of $B$. thuringiensis (Raymond et al., 2012), and this may be the case in many other bacterial pathogens (Schmid-Hempel and Frank, 2007). Public-goods-based virulence therefore challenges the key assumption of independent action, as cells must work together to successfully overcome barriers to infection, as recent experiments with $B$. thuringiensis have demonstrated (Cornforth et al., 2015). The fact that diversity is lost as infections proceed does not undermine the idea that bacteria can cooperate; rather it illustrates how the restricted invasibility of pathogens can facilitate a population genetic structure that favours cooperative interactions.

\section{Conflict of Interest}

The authors declare no conflict of interest. 


\section{Acknowledgements}

Raw experimental data are deposited in the SOM. This work was supported by a Natural Environment Research Fellowship award (to BR), and by BBSRC, partners of the ERASysBio+ initiative supported under the EU ERA-NET Plus scheme (to EvL). Thanks to Selina Brace, Francisco Úbeda, John Bryden and Vincent Jansen for comments on previous drafts of this work.

\section{Author contributions}

BR conceived the study and designed the experiments. EvL formulated the model and fitted the data. $\mathrm{BR}$ and EvL wrote the manuscript. BR, AM and SO performed the experiments and collected the data.

\section{References}

Akaike H. (1973). Information theory and an extension of the maximum likelihood principle. 2nd International Symposium on Information Theory Budapest: Publishing house of the Hungarian Academy of Sciences, 268-281.

Balmer O, Tanner M. (2011). Prevalence and implications of multiple-strain infections. Lancet Infect Dis 11: 868-878.

Bomberger JM, MacEachran DP, Coutermarsh BA, Ye S, O'Toole GA, Stanton BA. (2009). Long-distance delivery of bacterial virulence factors by Pseudomonas aeruginosa outer membrane vesicles. PLoS Pathog 5: e1000382.

Brest J, Greiner S, Boskovic B, Mernik M, Zumer V. (2006). Self-adapting control parameters in differential evolution: a comparative study on numerical benchmark problems. IEEE Transactions on Evolutionary Computation 10: 646-657.

Brockhurst MA, Hochberg ME, Bell T, Buckling A. (2006). Character displacement promotes cooperation in bacterial biofilms. Curr Biol 16: 2030-2034.

Brown SP, Cornell SJ, Sheppard M, Grant AJ, Maskell DJ, Grenfell BT et al. (2006). Intracellular demography and the dynamics of Salmonella enterica infections. PLoS Biol 4: e349.

Buchanan RL, Havelaar AH, Smith MA, Whiting RC, Julien E. (2009). The Key Events Dose-Response Framework: its potential for application to foodborne pathogenic microorganisms. Crit Rev Food Sci Nutr 49: 718-728.

Burnham KP, Anderson DR. (2002). Model Selection and Multi-Model Inference: A Practical Information-Theoretic Approach, 2nd edn. Springer-Verlag: New York.

Cespedes C, Said-Salim B, Miller M, Lo S-H, Kreiswirth BN, Gordon RJ et al. (2005). The clonality of Staphylococcus aureus nasal carriage. J Infect Dis 191: 444-452.

Collier FA, Elliot SL, Ellis RJ. (2005). Spatial variation in Bacillus thuringiensis/cereus populations within the phyllosphere of broad-leaved dock (Rumex obtusifolius) and surrounding habitats. FEMS Microbiol Ecol 54: $417-425$.

Cornforth DM, Matthews A, Brown SP, Raymond B. (2015). Bacterial cooperation causes systematic errors in pathogen risk assessment due to failure of the independent action hypothesis. PLoS Pathog 11: e10047705.

Damore JA, Gore J. (2012). Understanding microbial cooperation. J Theor Biol 299: 31-41.

Datta MS, Korolev KS, Cvijovic I, Dudley C, Gore J. (2013). Range expansion promotes cooperation in an experimental microbial metapopulation. Proc Natl Acad Sci USA 110: 7354-7359.

Diggle SP, Griffin AS, Campbell GS, West SA. (2007). Cooperation and conflict in quorum-sensing bacterial populations. Nature 450: 411-414.

Druett HA. (1952). Bacterial Invasion. Nature 170: 288-288.

Ellison AM. (1987). Effect of seed dimorphism on the density-dependent dynamics of experimental populations of Atriplex triangularis (Chenopodiaceae). Am J of Bot 74: 1280-1288.

Frank SA. (1998). Foundations of Social Evolution, 1st edn. Princeton University Press: Princeton, NJ, USA.

Garbutt J, Bonsall MB, Wright DJ, Raymond B. (2011). Antagonistic competition moderates virulence in Bacillus thuringiensis. Ecol Lett 14: 765-772.

Gilbert OM, Foster KR, Mehdiabadi NJ, Strassmann JE, Queller DC. (2007). High relatedness maintains multicellular cooperation in a social amoeba by controlling cheater mutants. Proc Natl Acad Sci USA 104: 8913-8917.

Gilbert OM, Strassmann JE, Queller DC. (2012). High relatedness in a social amoeba: the role of kin-discriminatory segregation. Proc Biol Sci 279: 2619-2624.

Grant AJ, Restif O, McKinley TJ, Sheppard M, Maskell DJ, Mastroeni P. (2008). Modelling within-host spatiotemporal dynamics of invasive bacterial disease. PLoS Biol 6: e74.

Griffin AS, West SA, Buckling A. (2004). Cooperation and competition in pathogenic bacteria. Nature 430: 1024-1027.

Haas CN. (1983). Estimation of risk due to low doses of microorganisms: a comparison of alternative methodologies. Am J Epidemiol 118: 573-582.

Hamilton WD. (1964). The genetical evolution of social behaviour. I. $J$ Theor Biol 7: 1-16.

Knell RJ, Begon M, Thompson DJ. (1996). Transmission dynamics of Bacillus thuringiensis infecting Plodia interpunctella: a test of the mass action assumption with an insect pathogen. Proc Biol Sci 263: 75-81.

Korolev KS, Xavier JB, Nelson DR, Foster KR. (2011). A quantitative test of population genetics using spatiogenetic patterns in bacterial colonies. Am Nat 178: 538-552.

Lecadet M-M, Blondel M-O, Ribier J. (1980). Generalized transduction in Bacillus thuringiensis var. berliner 1715 using bacteriophage CP-54Ber. J Gen Microbiol 121: 203-212.

Levert M, Zamfir O, Clermont O, Bouvet O, Lespinats S, Hipeaux MC et al. (2010). Molecular and evolutionary bases of within-patient genotypic and phenotypic diversity in Escherichia coli extraintestinal infections. PLoS Pathog 6: e1001125.

Margolis E, Yates A, Levin BR. (2010). The ecology of nasal colonization of Streptococcus pneumoniae, Haemophilus influenzae and Staphylococcus aureus: the role of competition and interactions with host's immune response. BMC Microbiol 10: 59.

Mitri S, Xavier JB, Foster KR. (2011). Social evolution in multispecies biofilms. Proc Natl Acad Sci USA 108: 10839-10846. 
Momeni B, Waite AJ, Shou W. (2013). Spatial selforganization favors heterotypic cooperation over cheating. Elife 2: e00960.

Moxon ER, Murphy PA. (1978). Haemophilus influenzae bacteremia and meningitis resulting from survival of a single organism. Proc Natl Acad Sci USA 75: 1534-1536.

Nadell CD, Bucci V, Drescher K, Levin SA, Bassler BL, Xavier JB. (2013). Cutting through the complexity of cell collectives. Proc $R$ Soc B 280: 20122770.

Nadell CD, Foster KR, Xavier JB. (2010). Emergence of spatial structure in cell groups and the evolution of cooperation. PLoS Comput Biol 6: e1000716.

Plaut RD, Kelly VK, Lee GM, Stibitz S, Merkel TJ. (2012). Dissemination bottleneck in a murine model of inhalational anthrax. Infect Immun 80: 3189-3193.

Prajsnar TK, Hamilton R, Garcia-Lara J, McVicker G, Williams A, Boots $\mathrm{M}$ et al. (2012). A privileged intraphagocyte niche is responsible for disseminated infection of Staphylococcus aureus in a zebrafish model. Cell Microbiol 14: 1600-1619.

Queller DC, Goodnight KF. (1989). Estimating relatedness using genetic markers. Evolution 43: 258-275.

Rankin DJ, Rocha EPC, Brown SP. (2011). What traits are carried on mobile genetic elements, and why? Heredity 106: $1-10$.

Raymond B, Johnston PR, Nielsen-LeRoux C, Lereclus D, Crickmore N. (2010a). Bacillus thuringiensis: an impotent pathogen? Trends Microbiol 18: 189-194.

Raymond B, Johnston PR, Wright DJ, Ellis RJ, Crickmore N, Bonsall MB. (2009). A mid-gut microbiota is not required for the pathogenicity of Bacillus thuringiensis to diamondback moth larvae. Environ Microbiol 11: 2556-2563.

Raymond B, West SA, Griffin AS, Bonsall MB. (2012). The dynamics of cooperative bacterial virulence in the field. Science 337: 85-88.

Raymond B, Wyres KL, Sheppard SK, Ellis RJ, Bonsall MB. (2010b). Environmental factors determining the epidemiology and population genetic structure of the Bacillus cereus group in the field. PLoS Pathog 6: e1000905.

Ross-Gillespie A, Gardner A, West SA, Griffin AS. (2007). Frequency dependence and cooperation: theory and a test with bacteria. Am Nat 170: 331-342.
Rubin LG. (1987). Bacterial colonization and infection resulting from multiplication of a single organism. Rev Infect Dis 9: 488-493.

Sachs JL, Mueller UG, Wilcox TP, Bull JJ. (2004). The evolution of cooperation. $Q$ Rev Biol 79: 135-160.

Schmid-Hempel P, Frank SA. (2007). Pathogenesis, virulence, and infective dose. PLoS Pathog 3: e147.

Schnepf E, Crickmore N, Van Rie J, Lereclus D, Baum J, Feitelson J et al. (1998). Bacillus thuringiensis and its pesticidal crystal proteins. Microbiol Mol Biol Rev 62: 775-806.

Slater FR, Bruce KD, Ellis RJ, Lilley AK, Turner SL. (2008). Heterogeneous selection in a spatially structured environment affects fitness tradeoffs of plasmid carriage in Pseudomonads. Appl Environ Microbiol 74: 3189-3197.

West SA, Buckling A. (2003). Cooperation, virulence and siderophore production in bacterial parasites. Proc Biol Sci 270: 37-44.

West SA, Griffin AS, Gardner A. (2007). Evolutionary explanations for cooperation. Curr Biol 17: R661-R672.

West SA, Griffin AS, Gardner A, Diggle SP. (2006). Social evolution theory for microorganisms. Nat Rev Microbiol 4: 597-607.

Zhou L, Slamti L, Nielsen-LeRoux C, Lereclus D, Raymond B. (2014). The social biology of quorum sensing in a naturalistic host pathogen system. Curr Biol 24: 2417-2422.

(c) (1) (2) () This work is licensed under a Creative Commons Attribution-NonCommercialShareAlike 4.0 International License. The images or other third party material in this article are included in the article's Creative Commons license, unless indicated otherwise in the credit line; if the material is not included under the Creative Commons license, users will need to obtain permission from the license holder to reproduce the material. To view a copy of this license, visit http://creativecommons.org/ licenses/by-nc-sa/4.0/

Supplementary Information accompanies this paper on The ISME Journal website (http://www.nature.com/ismej) 